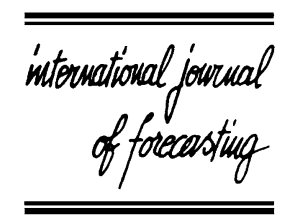

www.elsevier.com/locate/ijforecast

\title{
Extreme value theory and Value-at-Risk: Relative performance in emerging markets
}

\author{
Ramazan Gençay ${ }^{\mathrm{a}, \mathrm{b}, *}$, Faruk Selçuk ${ }^{\mathrm{c}, 1}$ \\ ${ }^{a}$ Department of Economics, University of Windsor, 401 Sunset Avenue, Windsor, Ontario, Canada N9B 3P4 \\ ${ }^{\mathrm{b}}$ HEC Genève, Faculte des Sciences Economiques et Sociales Uni Mail, Boulevard du Pont-d'Arve 40, CH-1211 Genève 4, Switzerland \\ ${ }^{\mathrm{c}}$ Department of Economics, Bilkent University, Bilkent 06533, Ankara, Turkey
}

\begin{abstract}
In this paper, we investigate the relative performance of Value-at-Risk (VaR) models with the daily stock market returns of nine different emerging markets. In addition to well-known modeling approaches, such as variance-covariance method and historical simulation, we study the extreme value theory (EVT) to generate VaR estimates and provide the tail forecasts of daily returns at the 0.999 percentile along with $95 \%$ confidence intervals for stress testing purposes. The results indicate that EVTbased VaR estimates are more accurate at higher quantiles. According to estimated Generalized Pareto Distribution parameters, certain moments of the return distributions do not exist in some countries. In addition, the daily return distributions have different moment properties at their right and left tails. Therefore, risk and reward are not equally likely in these economies. (C) 2004 International Institute of Forecasters. Published by Elsevier B.V. All rights reserved.
\end{abstract}

Keywords: Value-at-Risk; Financial risk management; Extreme value theory; Nonlinear tail forecasts

\section{Introduction}

Large changes are the most important of all. Not only for speculators,... but also for all students of the fundamental mechanisms of price changes (Mandelbrot, 2001).

Currency, liquidity, regulatory and interest rate risks in emerging markets ${ }^{2}$ have been the dominant

* Corresponding author. Department of Economics, Carleton University, 1125 Colonel By Drive, Ottawa, Ontario, K1S 5B6, Canada.

E-mail addresses: gencay@carleton.ca (R. Gençay), faruk@bilkent.edu.tr (F. Selçuk).

${ }^{1}$ This work was partially completed while Faruk Selçuk was a visiting associate professor at Colorado College, Colorado Springs, USA. engines of excessive volatility in the last 15 years. A recent example of high volatility in emerging economies and its effect on the rest of the world is the Asian crisis of $1997-1998 .^{3}$ In early 1997, the overall macroeconomic situation in far east Asia had deteriorated; it involved large current account

\footnotetext{
2 An emerging economy can be defined as a market economy with a small share in the world economy. Further, the currency of an emerging country can easily be traded but there is a widespread conception among market participants that the authorities in these economies can impose certain restrictions on the exchange rate and/ or capital flows any time. Also, political and economic stability in these economies are an exception, rather than a rule.

3 See Corsetti, Pesenti, and Roubini (1999) and references therein for a detailed account of these crises. A large number of studies are available online on Nouriel Roubini's Asian Crisis Homepage at www.stern.nyu.edu/ nroubini.
} 
deficits, increasing short-term debt, and most noticeably, large insolvent financial institutions who borrowed from abroad to finance the boom in real estate and equity investment. In June 1997, the Thai government declared its intention to abandon the policy of supporting/bailing out any financial institution. There was a strong speculative attack on the Thai baht and the authorities tried to defend the currency by increasing the short-term interest rates. Nevertheless, the attacks did not stop and the government let the baht float on July 2, 1997. This decision was a major turning point of the Asian crisis of 1997-1998. In 2 months, the currency depreciated $20 \%$. The currencies of other countries with similar economies to Thailand came under speculative attack as well. These countries were Malaysia, Indonesia and the Philippines. In September 1997, the baht was $42 \%$ below its January level, the rupiah $37 \%$ below and the ringgit $26 \%$ below.

The successful speculative attacks on these currencies had an immediate effect on neighboring countries. The Singaporean currency had lost $8 \%$ of its value in September 1997 as compared to January 1997. The speculative pressures continued in October 1997 in Taiwan and Hong Kong, forcing Taiwanese authorities to let their currency float. The largest economy in the area, Korea, was already suffering a series of bankruptcies and financial distress in the corporate sector, which directly affected the banking system in early 1997 . However, an early speculative attack did not occur in Korea. Nevertheless, the devaluations in other countries have put Korea in an uncompetitive position and in November 1997, the Korean currency depreciated $25 \%$ in 1 month. This was followed by devaluations in Taiwan and Singapore. Foreign banks refused to roll over the existing debt to these countries and the situation worsened. The financial panic led to a $40 \%$ currency collapse in Korea in 1 week in December and stopped only after a consortium of American, European and Japanese banks agreed to negotiate the roll over of the shortterm loans.

The turmoil did not stop in 1997. In January 1998, Indonesia was not able to roll over its short-term foreign debt and declared a moratorium. With severe macroeconomic conditions in Japan, an overall slowdown in economic growth in the world and a decline in commodity prices in general, the prospect for 1998 was very bleak. Suddenly, there was another currency crisis in Russia in August 1998, which resulted in domestic debt rescheduling and capital controls in that country. The impact of the Russian crash was felt across the world. In the United States, it triggered the collapse of the USD 100 billion Long Term Capital Management hedge fund and investors became extremely risk averse.

In Latin America, there was fear of concurrent devaluations, and the emerging market spreads over the U.S. T-bills increased sharply. Following the Russian crisis, the Brazilian government promised fiscal discipline and announced an austerity plan in late 1998. On January 6, 1999, a provincial governor who was a former president of Brazil, announced a 90-day moratorium on debt payments to the central government to protest the austerity plan. The move ignited an attack on the real (Brazil's currency) and there was a rapid capital outflow (about $\$ 1$ billion in 1 week). On January 13, the Central Bank decided to widen the band in which the real could be traded each day. The new band lasted only 2 days during which another 1 billion USD left the country. The next step was to let the real float freely and by February 3, the real was $32 \%$ below its January 13 value. In order to restore confidence, the Central Bank of Brazil raised the short-term interest rates from $29 \%$ to $39 \%$ and the capital flight slowed down.

The currencies of other Latin American countries had lost value against the US dollar between 1997 and March 1999: Chile's peso more than 13\%, Colombia's peso $22 \%$, Ecuador's sucre $43 \%$, Mexico's peso $30 \%$, Peru's sol 14\% and Venezuela's bolivar 18\%. Similar large movements were observed in equity markets as well. The year 2000 did not come with any comfort in emerging economies. Argentina was going through a deep recession for the last 3 years and the authorities were reluctant to take corrective measures in foreign exchange rates because of the currency board arrangement. A high inflation economy, Turkey, adopted a crawling peg regime (a tablita) to reduce inflation without having a "corrective devaluation" beforehand. Both countries were perceived as "next trouble spots" by international market participants and there was increasing concern of spillover effects. A first sign of discomfort emerged in November 2000 in Turkey. The rush of the IMF team to rescue the 
Turkish economy calmed down the markets temporarily. More recently, the decision to abandon the currency board arrangement in Argentina after months of social tension and the February 2001 crisis in Turkey during which the overnight interest rates hit (simple annual) $8000 \%$ proved once again that the volatility in emerging economies is an inherent part of the system.

The common lesson from these financial disasters is that billions of dollars can be lost because of poor supervision and management of financial risks. The Value-at-Risk (VaR) was developed in response to financial disasters of the 1990s and obtained an increasingly important role in market risk management. The VaR summarizes the worst loss over a target horizon with a given level of confidence. It is a popular approach because it provides a single quantity that summarizes the overall market risk faced by an institution. ${ }^{4}$

In a VaR context, precise prediction of the probability of an extreme movement in the value of a portfolio is essential for both risk management and regulatory purposes. By their very nature, extreme movements are related to the tails of the distribution of the underlying data generating process. Several tail studies which followed the pioneering work by Mandelbrot (1963a, 1963b) indicate that most financial time series are fat-tailed. ${ }^{5}$ Although these findings necessitate a definition of what is meant by a fat-tailed distribution, there is no unique definition of fat-tailedness (heavy-tailness) of a distribution in the lature. ${ }^{6}$ In this study, we consider a distribution to be fat-tailed if a power decay of the density function is observed in the tails. Accordingly, an exponential decay or a finite endpoint at the tail (the density

\footnotetext{
4 See Dowd (1998), Duffie and Pan (1997) and Jorion (1997) for more details on the VaR methodology. For the regulatory roots of the VaR, see Basel (1996).

5 See, for example, Boothe and Glassman (1987), Dacorogna, Gençay, Müller, Olsen, and Pictet (2001), Dacorogna, Pictet, Müller, and de Vries (2001), Danielsson and de Vries (1997), Ghose and Kroner (1995), Hauksson, Dacorogna, Domenig, Müller, and Samorodnitsky (2000), Hols and de Vries (1991), Koedijk, Schafgans, and de Vries (1990), Levich (1985), Loretan and Phillips (1994), Müller, Dacorogna, and Pictet (1998), Mussa (1979), and Pictet, Dacorogna, and Müller (1998).

${ }^{6}$ See Embrechts, Kluppelberg, and Mikosch (1997, Ch.2 and 8) for a detailed discussion.
}

reaching zero before a finite quantile) is treated as thin-tailed. ${ }^{7}$

In order to model fat-tailed distributions, the lognormal distribution, generalized error distribution and mixtures of normal distributions are suggested in many studies. However, these distributions are thintailed according to our definition since the tails of these distributions decay exponentially, although they have excess kurtosis over the normal distribution. In some practical applications, these distributions may fit the empirical distributions up to moderate quantiles but their fit deteriorates rapidly at high quantiles (at extremes).

This paper studies the nonlinear estimation and forecasting of the tails of return distributions in emerging markets. Instead of forcing a single distribution for the entire sample, it is possible to investigate only the tails of the return distributions using limit laws, given that only the tails are important for extreme values. Furthermore, the parametric modeling of the tails is convenient for the extrapolation of probability assignments to the quantiles even higher than the most extreme observation in the sample. One such approach is the extreme value theory (EVT) which provides a formal framework in which to study the tail behavior of the fattailed distributions.

The EVT stemming from statistics has found many applications in structural engineering, oceanography, hydrology, pollution studies, meteorology, material strength, highway traffic and many others. ${ }^{8}$ The link between the EVT and risk management is that EVT methods fit extreme quantiles better than the conventional approaches for heavy-tailed data. ${ }^{9}$ The EVT approach is also a convenient framework for the separate treatment of the tails of a distribution, which

\footnotetext{
${ }^{7}$ Although the fourth moment of an empirical distribution (sample kurtosis) is sometimes used to decide on whether an empirical distribution is heavy-tailed or not, this measure might be misleading. For example, the uniform distribution has excess kurtosis over the normal distribution but it is thin-tailed according to our definition.

${ }^{8}$ For an in-depth coverage of EVT and its applications in finance and insurance, see Embrechts et al. (1997), McNeil (1998), Reiss and Thomas (1997) and Teugels and Vynckier (1996).

9 See Embrechts (2000a) and Embrechts, Resnick, and Samorodnitsky (1998) for the efficiency of EVT as a risk management tool.
} 
allows for asymmetry. Considering the fact that most financial return series are asymmetric (Levich, 1985; Mussa, 1979), the EVT approach is advantageous over models which assume symmetric distributions such as $t$-distributions, normal distributions, $\mathrm{ARCH}$, GARCH-like distributions except E-GARCH which allows for asymmetry (Nelson, 1991).

Our results indicate that the daily return distributions have different moment restrictions at their left and right tails. Therefore, the risk and reward are not equally likely in these economies. Estimates of left and right tails at the 0.999 percentile along with $95 \%$ confidence intervals show that it is possible to observe over $10 \%$ losses in 1 day in all emerging markets. Model comparisons indicate that the GPD model clearly dominates others in terms of VaR forecasting at the 99th and higher quantiles. We conclude that the GPD and the extreme value theory are an indispensable part of risk management in general and the VaR calculations in particular, in emerging markets.

This paper is structured as follows. In Section 2, the extreme value theory and a review of some common tools of preliminary data analysis are covered. Section 3 presents the descriptive statistics and tail estimation. Section 4 reviews some popular approaches in VaR estimation and compares the relative performance of different models in terms of VaR violation ratios. We conclude afterwards.

\section{Extreme value theory}

Extreme value theory is a powerful and yet fairly robust framework in which to study the tail behavior of a distribution. Even though extreme value theory has previously found large applicability in climatology and hydrology, there have also been a number of extreme value studies in the finance literature in recent years. de Haan, Jansen, Koedijk, and de Vries (1994) study the quantile estimation using extreme value theory. McNeil $(1997,1998)$ study the estimation of the tails of loss severity distributions and the estimation of the quantile risk measures for financial time series using extreme value theory. Embrechts et al. (1998) overview the extreme value theory as a risk management tool. Müller et al. (1998) and Pictet et al. (1998) study the probability of exceedances for the foreign exchange rates and compare them with the GARCH and HARCH models. Embrechts (1999, 2000a) studies the potentials and limitations of the extreme value theory. McNeil (1999) provides an extensive overview of the extreme value theory for risk managers. McNeil and Frey (2000) study the estimation of tail-related risk measures for heteroskedastic financial time series. Embrechts (2000b), Embrechts et al. (1997) are a comprehensive source of the extreme value theory to the finance and insurance literature.

In the following section, we present the parametric framework for our study. Within the EVT context, there are two approaches to study the extremal events. One of them is the direct modeling of the distribution of minimum or maximum realizations. The other one is modeling the exceedances of a particular threshold.

\subsection{Fisher-Tippett theorem}

The normal distribution is the important limiting distribution for sample averages as summarized in a central limit theorem. Similarly, the family of extreme value distributions is the one to study the limiting distributions of the sample extrema. This family can be presented under a single parameterization known as the generalized extreme value distribution (GEV). The theory deals with the convergence of maxima. Suppose that $X_{t}, t=1,2, \ldots, n$ is a sequence of independently and identically distributed $^{10}$ random variables with a common distribution function $F(x)=\operatorname{Pr}\left\{X_{t} \leq x\right\}$ which has mean (location parameter) $\mu$ and variance (scale parameter) $\sigma^{2} .^{11}$ Denote the sample maxima ${ }^{12}$ of $X_{t}$ by $M_{1}=X_{1}$, $M_{n}=\max \left(X_{1}, \ldots, X_{n}\right), n \geq 2$ and let $\Re$ denote the real line. Given a sequence of $c_{n}>0, d_{n} \in \mathfrak{R}$ and some non-degenerate distribution function $H$ such

\footnotetext{
10 The assumption of independence can be easily dropped and the theoretical results follow through. See McNeil (1997). The assumption of identical distribution is for convenience and can also be relaxed.

${ }^{11}$ For convenience, we will assume that $\mu=0$ and $\sigma^{2}=1$ in this section.

${ }^{12}$ The sample maxima is $\min \left(X_{1}, \ldots, X_{n}\right)=-\max \left(-X_{1}, \ldots\right.$, $-X_{n}$ ).
} 
that $c_{n}^{-1}\left(M_{n}-d_{n}\right) \stackrel{d}{\rightarrow} H$, then $H$ belongs to one of the following three families of distributions:

Gumbel : $\Lambda(x)=\exp \left(-\exp ^{-x}\right), \quad x \in \Re$

Fréchet : $\Phi_{\alpha}(x)= \begin{cases}0, & x \leq 0 \\ \exp \left(-x^{-\alpha}\right), & x>0, \quad \alpha>0\end{cases}$

Weibull : $\Psi_{\alpha}(x)= \begin{cases}\exp \left[-\left(-x^{-\alpha}\right)\right], & x \leq 0, \quad \alpha<0 \\ 1, & x>0 .\end{cases}$

The Fisher and Tippett (1928) theorem ${ }^{13}$ suggests that the asymptotic distribution of the maxima belongs to one of the three distributions above, ${ }^{14}$ regardless of the original distribution of the observed data. ${ }^{15}$

Fréchet and Weibull distributions attain the shape of a Gumbel distribution when the tail index parameter $\alpha$ goes to $\infty$ and $-\infty$, respectively. By taking the reparameterization $\xi=1 / \alpha$, due to Jenkinson (1955) and von Mises (1936), Fréchet, Weibull and Gumbel distributions can be represented in a unified model with a single parameter. This representation is known as the generalized extreme value distribution (GEV)

$H_{\xi}(x)= \begin{cases}\exp \left\{-(1+\xi x)^{-1 / \xi}\right\}, & \text { if } \xi \neq 0,1+\xi x>0 \\ \exp \{-\exp (-x)\}, & \text { if } \xi=0\end{cases}$

where $\xi=1 / \alpha$ is a shape parameter and $\alpha$ is the tail index.

The class of distributions of $F(x)$ where the Fisher-Tippett theorem holds is quite large. ${ }^{16}$ One

\footnotetext{
13 The first formal proof of the Fisher-Tippett theorem is given in Gnedenko (1943).

14 In conventional statistics, a Weibull distribution function $F_{\alpha}(x)$ is defined as $F_{\alpha}(x)=1-e^{-x \alpha}$ for $x>0$. The Weibull distribution function $\Psi_{\alpha}(x)$ above is concentrated on $(-\infty, 0)$ and it is $\Psi_{\alpha}(x)=1-F_{\alpha}(-x)$ for $x<0 . F_{\alpha}(x)$ and $\Psi_{\alpha}(x)$ have completely different extremal behavior. In the extreme value theory literature, $\Psi_{\alpha}(x)$ is referred to as the Weibull distribution. See Embrechts et al. (1997, Ch. 3).

15 The interested reader will find the full development of the theory in de Haan (1990) and Leadbetter, Lindgren, and Rootzén (1983).

16 Embrechts (1999) Embrechts et al. (1997, 1998) and McNeil (1997, 1999), have excellent discussions of the theory behind the extreme value distributions from the risk management perspective.
}

of the conditions is that $F(x)$ has to be in the domain of attraction for the Fréchet distribution ${ }^{17}$ $(\xi>0)$ which in general holds for the financial time series. Gnedenko (1943) shows that if the tail of $F(x)$ decays like a power function, then it is in the domain of attraction for the Fréchet distribution. The class of distributions whose tails decay like a power function is large and includes the Pareto, Cauchy, Student's $t$-test and mixture distributions. These distributions are the well-known heavy-tailed distributions.

\subsection{Number of exceedances over a threshold}

In general, we are not only interested in the maxima of observations, but also in the behavior of large observations which exceed a high threshold. One method of extracting extremes from a sample of observations, $X_{t}, t=1,2, \ldots, n$ with a distribution function $F(x)=\operatorname{Pr}\left\{X_{t} \leq x\right\}$ is to take the exceedances over a predetermined, high threshold $u$. An exceedance of a threshold $u$ occurs when $X_{t}>u$ for any $t$ in $t=1,2, \ldots, n$. An excess over $u$ is defined by $y=X_{i}-u{ }^{18}$

Given a high threshold $u$, the probability distribution of excess values of $X$ over threshold $u$ is defined by

$$
F_{u}(y)=\operatorname{Pr}\{X-u \leq y \mid X>u\}
$$

which represents the probability that the value of $X$ exceeds the threshold $u$ by at most an amount $y$ given that $X$ exceeds the threshold $u$. This conditional probability may be written as

$$
\begin{aligned}
F_{u}(y) & =\frac{\operatorname{Pr}\{X-u \leq y, X>u\}}{\operatorname{Pr}(X>u)} \\
& =\frac{F(y+u)-F(u)}{1-F(u)} .
\end{aligned}
$$

Since $x=y+u$ for $X>u$, we have the following representation

$F(x)=[1-F(u)] F_{u}(y)+F(u)$.

Notice that this representation is valid only for $X>u$.

A theorem by Balkema and de Haan (1974) and Pickands (1975) shows that for sufficiently high threshold $u$, the distribution function of the excess

\footnotetext{
17 See Falk, Hüssler, and Reiss (1994).

18 This is also referred to as the Peaks-over-Threshold (POT).
} 
may be approximated by the generalized Pareto distribution (GPD) because as the threshold gets large, the excess distribution $F_{u}(y)$ converges to the GPD. The GPD in general is defined as

$G_{\xi, \sigma, v}(x)= \begin{cases}1-\left(1+\xi \frac{x-v}{\sigma}\right)^{-1 / \xi} & \text { if } \xi \neq 0 \\ 1-e^{-(x-v) / \sigma} & \text { if } \xi=0,\end{cases}$

with

$x \in \begin{cases}{[v, \infty],} & \text { if } \xi \geq 0 \\ {[v, v-\sigma / \xi],} & \text { if } \xi<0\end{cases}$

where $\xi=1 / \alpha$ is the shape parameter, $\alpha$ is the tail index, $\sigma$ is the scale parameter, and $v$ is the location parameter. When $v=0$ and $\sigma=1$, the representation is known as the standard GPD. There is a simple relationship between the standard GDP $G_{\xi}(x)$ and $H_{\xi}(x)$ such that $G_{\xi}(x)=1+\log H_{\xi}(x)$ if $\log H_{\xi}(x)>-1$.

The GPD embeds a number of other distributions. When $\xi>0$, it takes the form of the ordinary Pareto distribution. This particular case is the most relevant for financial time series analysis since it is a heavytailed one. For $\xi>0, E\left[X^{k}\right]$ is infinite for $k>1 / \xi$. For instance, the GPD has an infinite variance for $\xi=0.5$ and, when $\xi=0.25$, it has an infinite fourth moment. For the security returns or high frequency foreign exchange returns, the estimates of $\xi$ are usually less than 0.5, implying that the returns have finite variance (Dacorogna, Gençay, et al., 2001). When $\xi=0$, the GPD corresponds to the exponential distribution and it is known as a Pareto II type distribution for $\xi<0$.

The importance of the Balkema and de Haan (1974) and Pickands (1975) results is that the distribution of excesses may be approximated by the GPD by choosing $\xi$ and setting a high threshold $u$. The GPD model can be estimated with the maximum likelihood method. For $\xi>-0.5$, Hosking and Wallis (1987) present evidence that maximum likelihood regularity conditions are fulfilled and the maximum likelihood estimates are asymptotically normally distributed. Therefore, the approximate standard errors for the estimator of $\xi$ can be obtained through maximum likelihood estimation.

For the tail estimation, recall from Eq. (3) that

$F(x)=[1-F(u)] F_{u}(y)+F(u)$.
Since $F_{u}(y)$ converges to the GPD for sufficiently large $u$, and since $x=y+u$ for $X>u$, we have

$F(x)=[1-F(u)] G_{\xi, \sigma, u}(x-u)+F(u)$.

After determining a high threshold $u$, the last term on the right hand side can be determined by $\left(n-n_{u}\right) / n$ where $n_{u}$ is the number of exceedances and $n$ is the sample size. As a result, we have the following estimator

$$
\begin{aligned}
\hat{F}(x) & =\left(1-\frac{n-n_{u}}{n}\right) G_{\hat{\xi}, \hat{\sigma}, u}(x-u)+\frac{n-n_{u}}{n} \\
& =\frac{n_{u}}{n} G_{\hat{\xi}, \hat{\sigma}, u}(x-u)+\frac{n-n_{u}}{n} \\
& =1+\frac{n_{u}}{n}\left(G_{\hat{\xi}, \hat{\sigma}, u}(x-u)-1\right) .
\end{aligned}
$$

Therefore, the tail estimator becomes

$$
\begin{aligned}
& \hat{F}(x)=1-\frac{n_{u}}{n}\left(1+\hat{\xi} \frac{x-u}{\hat{\sigma}}\right)^{-1 / \hat{\xi}} \text { given that } \\
& G_{\xi, \sigma, u}(x)=1-\left(1+\xi \frac{x-u}{\sigma}\right)^{-1 / \xi}
\end{aligned}
$$

where $\hat{\xi}$ and $\hat{\sigma}$ are the maximum likelihood estimators. Notice that the estimator in Eq. (6) is valid only for $X>u$.

\subsection{Preliminary data analysis}

In the extreme value theory and applications, the QQ-plot (quantile-quantile plot) is typically plotted against the exponential distribution (i.e. a distribution with a thin-sized tail) to measure the fat-tailness of a distribution. If the data is from an exponential distribution, the points on the graph would lie along a positively sloped straight line. If there is a concave presence, this would indicate a fat-tailed distribution, whereas a convex departure is an indication of a shorttailed distribution. ${ }^{19}$

19 If the sample is a realization from a distribution which has the same form as the reference distribution but with different scale and/or location parameters, the QQ-plot is still linear. In this case, the intercept of the QQ-plot indicates the location parameter while the scale parameter determines the slope. 
A second tool is the sample mean excess function (MEF) which is defined by

$e_{n}(u)=\frac{\sum_{i=1}^{n}\left(X_{i}-u\right)}{\sum_{i=1}^{n} I_{\left\{X_{i}>u\right\}}}$

where $I$ is an indicator function. The MEF is the sum of the excesses over the threshold $u$ divided by the number of data points which exceeds the threshold $u$. It is an estimate of the mean excess function which describes the expected overshoot of a threshold once an exceedance occurs. If the empirical MEF is a positively sloped straight line above a certain threshold $u$, it is an indication that the data follows the GPD with a positive shape parameter $\xi$. On the other hand, exponentially distributed data would show a horizontal MEF while short-tailed data would have a negatively sloped line.

Another tool in threshold determination is the Hill-plot. ${ }^{20}$ Hill (1975) proposed an estimator of $\xi$ when $\xi>0$ (Fréchet Case). By ordering the data with respect to their values as $X_{1, n}, X_{2, n}, X_{3, n}, \ldots, X_{n, n}$ where $X_{1, n} \geq X_{2, n} \geq X_{1, n} \geq \ldots \geq X_{n, n}$, the Hill's Estimator of the tail index $\xi$ is

$\hat{\xi}=\frac{1}{k} \sum_{i=j}^{k} \ln X_{j, n}-\ln X_{k, n}$

where $k \rightarrow \infty$ is upper order statistics (the number of exceedances), ${ }^{21} n$ is the sample size, and $\alpha=1 / \xi$ is the tail index. A Hill-plot is constructed such that estimated $\xi$ is plotted as a function of $k$ upper order statistics or the threshold. A threshold is selected from the plot where the shape parameter $\xi$ is fairly stable.

The Hill estimator is proven to be a consistent estimator of $\xi=1 / \alpha$ for fat-tailed distributions in Mason (1982). The conditions on $k$ and $n$ for weak consistency of the Hill's estimator are given in Mason (1982) and Rootzén, Leadbetter, and de Haan (1992). Deheuvels, Hausler, and Mason (1988) investigate the

\footnotetext{
20 See Embrechts et al. (1997) for a detailed discussion and several examples of the Hill-plot.

${ }^{21}$ The $i$ th element from the ordered sample, $X_{i, n}$ is called $i$ th upper order statistic.
}

conditions for the strong consistency of the Hill's estimator. From Hall (1982) and Goldie and Smith (1987), it follows that $(\hat{\xi}-\xi) k^{1 / 2}$ is asymptotically normally distributed with zero mean and variance $\xi^{2}$.

A difficulty of the Hill's estimator is the ambiguity of the value of threshold parameter, $k$. In threshold determination, we face a trade off between bias and variance. If we choose a low threshold, the number of observations (exceedances) increases and the estimation becomes more precise. However, choosing a low threshold also introduces some observations from the center of the distribution and the estimation becomes biased. While the estimates of $\xi$ based on a few largest observations are highly sensitive to the number of observations used, the estimates based on many elements from the top of the ordering are biased. ${ }^{22}$ Therefore, a careful combination of several techniques, such as the QQ-plot, the Hill-plot and the MEF should be considered in threshold determination.

\section{Data description and tail analysis}

Dynamics of financial markets in emerging countries show substantial differences as compared to developed economies. These markets experience larger "financial earthquakes" than developed economies, and can be labeled as "markets with many fault lines". Since a significant portion of total savings in developed economies are invested in emerging markets by hedge and mutual funds, the implications of these dynamics are not confined with the residents of emerging market countries. ${ }^{23}$ Therefore, a careful investigation of the market dynamics in these economies would benefit investors at large by increasing the investor awareness. ${ }^{24}$

\footnotetext{
22 Danielsson, de Haan, Pend, and de Vries (2001) propose a standardized procedure for choosing an optimal $k$ value.

23 The net portfolio investment in emerging markets by industrialized countries was USD 58.3 billion in 2000. The historical record is USD 109.9 billion in 1994. Source: IMF, International Financial Statistics.

${ }^{24}$ Foreign investors may face completely different financial circumstances in emerging economies than they have in their home country. To point this out, Dornbush (2001) refers to the catchy title of an article written in the early 1980s about the debt crisis in Latin American economies: "We are not in Kansas anymore..." (Diaz, 1984).
} 
In order to investigate the risk and reward dynamics in emerging economies, we collected daily stock market data from Argentina, Brazil, Hong Kong, Indonesia, Korea, Mexico, Singapore, Taiwan and Turkey. Descriptive statistics of daily returns are presented in Table 1 . The daily returns are defined as

$$
\begin{aligned}
r_{i, t} & =\log \left(x_{i, t} / x_{i, t-1}\right) \times 100 \\
& =\left(\log x_{i, t}-\log x_{i, t-1}\right) \times 100
\end{aligned}
$$

where $x_{i, t}$ is the daily closing value of the stock market index in country $i$ on day $t$.

The highest averages of the daily returns are in Brazil (0.37\%), Turkey (0.22\%) and Mexico (0.06\%). These daily return rates imply (annualized compound) returns of $161 \%$ in Brazil, $77 \%$ in Turkey and $17 \%$ in Mexico. The unusual average positive rate of return in Brazil and Turkey is possibly a product of high inflation rates in these countries. The annual rate of inflation (consumer prices) in Brazil was over 2000\% per year in 1993 and 1994 before it was stabilized under $10 \%$. The annual rate of inflation (consumer prices) in Turkey fluctuated between $60 \%$ and $120 \%$ during 1988-1999. Not surprisingly, Brasil and Turkey also have the highest standard deviations $(3.2 \%$ and $3 \%$ ) of the daily stock returns. The lowest averages of the daily log returns are in Korea $(-0.02 \%)$ and Argentina $(0.01 \%)$. Among these two, Korea stands out with a high standard deviation of $2.1 \%$.

According to the sample kurtosis estimates, the daily rate of returns are far from being normally distributed. The lowest kurtosis estimates are 7.4 (Korea) and 8.0 (Turkey), while the highest estimates are 61.2 (Singapore) and 36.6 (Hong Kong). Based on the sample kurtosis estimates, it may be argued that the return distributions in all the markets are fattailed. We also studied the QQ-plots of returns against the exponential distribution for each country. These plots confirm that the return distributions have fat tails.

The sample skewness shows that the daily returns have a symmetric distribution only in Argentina. In all other countries, the returns have either positive or negative skewness. The sample skewnesses are negative in Hong Kong $(-1.43)$, Indonesia $(-1.29)$, Mexico $(-0.20)$ and Singapore $(-2.21)$. This indicates that the asymmetric tail extends more towards negative values than positive ones. Positive skewness in other countries ranges from 0.09 (Taiwan) to 0.54 (Brazil).

Table 1 also shows the highest and lowest 1 day return from each country. The highest 1 day positive returns are in Turkey (30.5\%), Brazil $(28.8 \%)$ and Taiwan (19.9\%). The highest 1 day losses are in Hong Kong (40.5\%), Singapore (29.2\%) and Turkey (19.8\%).

Table 1

Descriptive statistics of the daily returns, $\log \left(x_{t} / x_{t-1}\right) \times 100$, from nine emerging stock markets

\begin{tabular}{llrlrrrrrr}
\hline & $n$ & Mean & Std & \multicolumn{1}{c}{ Ku } & \multicolumn{1}{c}{ Sk } & Min & Max & Low & High \\
\hline Argentina & 1935 & 0.01 & 0.019 & 9.19 & 0.00 & -13.6 & 11.9 & -3.0 & 2.7 \\
Brazil & 2086 & 0.37 & 0.030 & 10.56 & 0.54 & -17.2 & 28.8 & -4.3 & 5.3 \\
Hong Kong & 7305 & 0.04 & 0.020 & 36.64 & -1.43 & -40.5 & 17.2 & -2.9 & 2.8 \\
Indonesia & 2085 & 0.04 & 0.011 & 16.92 & -1.29 & -11.8 & 6.7 & -1.5 & 1.6 \\
Korea & 2868 & -0.02 & 0.021 & 7.36 & 0.27 & -12.5 & 14.6 & -3.3 & 3.5 \\
Mexico & 1453 & 0.06 & 0.020 & 10.90 & -0.20 & -15.0 & 13.3 & -3.0 & 3.2 \\
Philippines & 1076 & -0.02 & 0.015 & 6.78 & 0.10 & -7.9 & 9.1 & -2.5 & 2.4 \\
Singapore & 3910 & 0.04 & 0.014 & 61.25 & -2.21 & -29.2 & 15.5 & -1.8 & 1.9 \\
Taiwan & 7305 & 0.04 & 0.019 & 11.82 & 0.09 & -19.7 & 19.9 & -2.9 & 2.9 \\
Turkey & 3223 & 0.22 & 0.032 & 8.04 & 0.15 & -19.8 & 30.5 & -4.8 & 5.2 \\
\hline
\end{tabular}

$n=$ Sample size; Mean=Sample mean; Std=Standard deviation; $\mathrm{Ku}=$ Kurtosis; Sk=Skewness; Min= Minimum observed daily return; Max = Maximum observed daily return; Low = Daily return corresponding to 5th percentile; High=Daily return corresponding to 95 th percentile. Sample periods: Argentina: August 2, 1993, to December 29, 2000; Brazil: January 1, 1993 to December 29, 2000; Hong Kong: January 1, 1973 to December 29, 2000; Indonesia: January 4, 1993 to December 29, 2000; Korea: January 3, 1990 to December 29, 2000; Mexico: June 6, 1995 to December 29, 2000; Singapore: January 4, 1985 to December 29, 2000; Taiwan: January 1, 1973 to December 29, 2000; Turkey: January 8, 1988 to December 29, 2000. Data source: Datastream. 
Table 2

Threshold percentage returns, corresponding empirical quantiles and the number of exceedances

\begin{tabular}{|c|c|c|c|c|c|c|}
\hline & \multicolumn{3}{|l|}{ Lower tail } & \multicolumn{3}{|l|}{ Upper tail } \\
\hline & Threshold (\%) & Quantile & Exceedances & Threshold (\%) & Quantile & Exceedances \\
\hline Argentina & -2.7 & 6.7 & 129 & 1.7 & 87.2 & 247 \\
\hline Brazil & -3.8 & 7.0 & 130 & 8.0 & 98.5 & 32 \\
\hline Hong Kong & -7.0 & 0.6 & 41 & 2.0 & 90.3 & 706 \\
\hline Indonesia & -1.0 & 10.0 & 21 & 1.4 & 93.3 & 140 \\
\hline Korea & -3.5 & 4.5 & 130 & 7.0 & 99.0 & 20 \\
\hline Mexico & -3.0 & 5.0 & 69 & 4.1 & 97.1 & 41 \\
\hline Philippines & -4.0 & 1.8 & 19 & 3.3 & 97.8 & 24 \\
\hline Singapore & -2.5 & 2.6 & 101 & 2.0 & 95.0 & 179 \\
\hline Taiwan & -6.5 & 0.6 & 41 & 6.0 & 99.3 & 48 \\
\hline Turkey & -9.0 & 1 & 28 & 8.3 & 98.8 & 38 \\
\hline
\end{tabular}

\subsection{Tail estimation}

The crucial step in estimating the parameters of the GPD is the determination of a threshold value $u$. For each country, we examined the threshold at which the sample mean excess function has a positive slope. A visual inspection of the QQ-plots was also helpful to determine a range for the threshold values. We also used the Hill estimator for each country and compared it with the mean excess function and the QQ-plots. The selected threshold percentage return, corresponding sample quantile and the number of exceedances are given in Table 2.

The maximum likelihood estimates of the tail index $\xi$ and the scale parameter $\sigma$ with corresponding standard errors are presented in Table $3 .{ }^{25}$ The estimated tail index values range between -0.18 (Indonesia, right tail) and 0.60 (Taiwan, left tail). High values of the estimated tail index for the left tail in all emerging markets (except Korea) is an indication that these markets experienced severe crashes. The results also indicate that the right tail and the left tail of the stock return distributions have different moment properties.

For Hong Kong, Mexico, Singapore and Taiwan, the left tail index is over 0.40 . This is an indication of the high risk associated with these markets. Since the right tail index is less than the left tail index, we can conclude that risk and reward are not equally likely in

\footnotetext{
25 The results are obtained using the EVIM (Extreme Value Analysis in MATLAB) software. See Gençay, Selçuk, and Ulugülyağc1 (2001) for more details.
}

these economies. On the other hand, Argentina, Brazil, Korea and Turkey have higher estimates of the right tail index than of the left tail index. Therefore, high positive returns are more likely than similar losses in these economies.

The positive stock return distribution in Brazil, Taiwan and Turkey may not have a finite second moment since the estimated $\xi$ is around 0.50 for these countries. On the other hand, the positive stock return distribution in Argentina, Hong Kong and Singapore have the first three moments but may not have the fourth moment since the estimated $\xi$ is around 0.25 . For the left tail, we have a different picture. The negative stock return distribution may not have a finite second moment in Hong Kong, Mexico, Singapore Iand Taiwan, whereas the first four moments of the negative stock return distribution seems to exist in Argentina, Brazil and Turkey.

Table 3

Maximum Likelihood Estimates (MLE) of the parameters of the Generalized Pareto Distribution (GPD)

\begin{tabular}{|c|c|c|c|c|c|c|c|c|}
\hline & \multicolumn{4}{|c|}{ Lower tail } & \multicolumn{4}{|c|}{ Upper tail } \\
\hline & $\overline{\hat{\xi}}$ & $\operatorname{se}(\xi)$ & $\hat{\sigma}$ & $\overline{\operatorname{se}(\sigma)}$ & $\overline{\hat{\xi}}$ & $\operatorname{se}(\xi)$ & $\hat{\sigma}$ & $\operatorname{se}(\sigma)$ \\
\hline Argentina & 0.20 & 0.01 & 1.1 & 0.2 & 0.27 & 0.08 & 1.1 & 0.1 \\
\hline Brazil & 0.15 & 0.12 & 1.8 & 0.3 & 0.48 & 0.26 & 1.5 & 0.5 \\
\hline Hong Kong & 0.48 & 0.22 & 1.6 & 0.4 & 0.24 & 0.05 & 1.1 & 0.0 \\
\hline Indonesia & 0.32 & 0.09 & 0.6 & 0.1 & 0.18 & 0.10 & 0.5 & 0.1 \\
\hline Korea & 0.03 & 0.11 & 1.5 & 0.2 & 0.20 & 0.32 & 1.3 & 0.5 \\
\hline Mexico & 0.42 & 0.18 & 1.0 & 0.2 & 0.30 & 0.22 & 1.1 & 0.3 \\
\hline Philippines & 0.44 & 0.37 & 0.5 & 0.2 & -0.09 & 0.20 & 1.5 & 0.4 \\
\hline Singapore & 0.48 & 0.14 & 1.0 & 0.2 & 0.26 & 0.09 & 0.9 & 0.1 \\
\hline Taiwan & 0.60 & 0.26 & 0.7 & 0.2 & 0.53 & 0.25 & 1.1 & 0.3 \\
\hline Turkey & 0.22 & 0.27 & 1.6 & 0.5 & 0.59 & 0.26 & 1.1 & 0.3 \\
\hline
\end{tabular}


Table 4

Estimated daily percent returns at 0.999 percentile with $95 \%$ confidence levels

\begin{tabular}{lrrrrrrr}
\hline & \multicolumn{2}{l}{ Lower tail } & & \multicolumn{2}{l}{ Upper tail } \\
\cline { 2 - 3 } \cline { 7 - 8 } & $\mathrm{CI}_{\text {lower }}$ & Return & $\mathrm{CI}_{\text {upper }}$ & & $\mathrm{CI}_{\text {lower }}$ & Return & $\mathrm{CI}_{\text {upper }}$ \\
\hline Argentina & -8.2 & -10.1 & -15.1 & 8.9 & 11.6 & 17.5 \\
Brazil & -12.0 & -14.7 & -22.1 & & 13.2 & 16.7 & 34.3 \\
Hong Kong & -9.9 & -11.4 & -14.5 & 9.8 & 11.3 & 13.7 \\
Indonesia & -5.7 & -7.6 & -12.1 & & 4.1 & 4.8 & 6.6 \\
Korea & -8.5 & -9.5 & -12.0 & 8.9 & 10.1 & 14.0 \\
Mexico & -8.7 & -12.2 & -22.6 & 8.1 & 10.0 & 20.0 \\
Philippines & -5.4 & -6.8 & -11.9 & & 6.2 & 7.4 & 12.5 \\
Singapore & -7.7 & -10.1 & -16.4 & 6.7 & 8.1 & 11.0 \\
Taiwan & -7.9 & -8.7 & -10.5 & 8.4 & 9.6 & 12.4 \\
Turkey & -12.1 & -13.6 & -18.3 & & 12.1 & 14.3 & 22.5 \\
\hline
\end{tabular}

\subsection{Point predictions at the tails}

A portfolio manager is interested not only in expected return in a given market, but also expected extreme returns. A financial institution would likely see the possible change in the value of its balance sheet under extreme stress. This is possible by estimating a tail percentile through a GPD model. For each country, we obtained 0.999 percentile values (1 day in 4 years, since there are approximately 260 business days in each year) for left and right tails along with $95 \%$ confidence intervals based on the maximum likelihood method. The results are presented in Table 4. The estimated highest 1 day positive returns are in Brazil (16.7\%), Turkey (14.3\%) and Argentina (11.6\%). The highest estimated 1 day negative returns are Brazil (14.7\%), Turkey (13.6\%) and Mexico (12.2\%). Upper confidence intervals indicate that it is possible to observe over $10 \%$ loses in 1 day in all emerging markets, ranging from a low $10.5 \% 1$ day loss in Taiwan to a high $22.6 \%$ loss in Mexico. In comparison, the lower confidence intervals for the positive returns are less than $10 \%$ except in Brazil and Turkey.

\section{Modeling Value-at-Risk}

Let $r_{t}=\log \left(p_{t} / p_{t-1}\right)$ be the returns at time $t$ where $p_{t}$ is the price of an asset (or portfolio) at time $t$. The $\operatorname{VaR}_{t}(\alpha)$ at the $(1-\alpha)$ percentile is defined by

$\operatorname{Pr}\left(r_{t} \leq \operatorname{VaR}_{t}(\alpha)\right)=\alpha$ which calculates the probability that returns at time $t$ will be less than (or equal to) $\operatorname{VaR}_{t}(\alpha), \alpha$ percent of the time. ${ }^{26}$ The VaR is the maximum potential increase in value of a portfolio given the specifications of normal market conditions, time horizon and a level of statistical confidence. The VaR's popularity originates from the aggregation of several components of risk at firm and market levels into a single number.

The acceptance and usage of VaR has been spreading rapidly since its inception in the early 1990s. The VaR is supported by the Group of Ten banks, the Group of Thirty and the Bank for International Settlements and the European Union. The limitations of the VaR are that it may lead to a wide variety of results under a wide variety of assumptions and methods; it focuses on a single somewhat arbitrary point; it explicitly does not address exposure in extreme market conditions; and it is a statistical measure, not a managerial/economic one.

The methods used for VaR can be grouped under the parametric and nonparametric approaches. In this paper, we study the VaR estimation with extreme value theory (EVT) which is a parametric approach. The advantage of the EVT is that it focuses on the tails of the sample distribution when only the tails are important for practical purposes. Since fitting a single distribution to the entire sample imposes too much structure and our need here is the tails, we adopt the EVT framework which is what is needed to calculate the VaR. We compare six different models for estimating one period ahead return predictions in both tails of the return distribution at different tail quantiles. These models are the variance-covariance approach with normal distribution, variance-covariance approach with Student's $t$ distribution, historical simulation, adaptive generalized Pareto distribution (GPD) and nonadaptive GPD.

\subsection{Variance-covariance method}

The variance-covariance method is the simplest approach among the various models used to estimate the VaR. Let the sample of observations be denoted by $r_{t}, t=1,2, \ldots, n$ where $n$ is the sample size. Let us assume that $r_{t}$ follows a martingale process with

\footnotetext{
26 A typical value of $\alpha$ is $5 \%$ or $1 \%$.
} 
$r_{t}=\mu_{t}+\epsilon_{t}$ where $\epsilon_{t}$ has a distribution function $F$ with zero mean and variance, $\sigma_{t}^{2}$. The VaR in this case can be calculated as

$\operatorname{VaR}_{t}(\alpha)=\hat{\mu}_{t}+F^{-1}(\alpha) \hat{\sigma}_{t}$

where $F^{-1}(\alpha)$ is the $q$ th quantile $(q=1-\alpha)$ value of the unknown distribution function $F$. An estimate of $\mu_{t}$ and $\sigma_{t}^{2}$ can be obtained from the sample mean and the sample variance by

$\hat{\mu}_{t}=\frac{1}{n} \sum_{i=1}^{n} r_{i}, \quad \hat{\sigma}_{t}^{2}=\frac{1}{n-1} \sum_{i=1}^{n}\left(r_{i}-\hat{\mu}_{t}\right)^{2}$.

Although sample variance as an estimator of the standard deviation in variance-covariance approach is simple, it has drawbacks at high quantiles of a fattailed empirical distribution. The quantile estimates of the variance-covariance method for the right tail (left tail) are biased downwards (upwards) for high quantiles of a fat-tailed empirical distribution. Therefore, the risk is underestimated with this approach. Another drawback of this method is that it is not appropriate for asymmetric distributions. Despite these drawbacks, this approach is commonly used for calculating the VaR from holding a certain portfolio, since the $\mathrm{VaR}$ is additive when it is based on sample variance under the normality assumption.

Instead of the sample variance, the standard deviation in Eq. (11) can be estimated by a statistical model. Since financial time series exhibit volatility clustering, the ARCH (Engle, 1982) and GARCH (Bollerslev, 1986) are popular models for volatility modeling. ${ }^{27}$ If $r_{t}$ follows a $\operatorname{GARCH}(\mathrm{p}, \mathrm{q})$ model, then

$r_{t}=v_{t} \sqrt{h_{t}}$

where $v_{t}$ is a Gaussian white noise with constant variance $\sigma_{v}^{2}=1$, and $h_{t}$ is

$h_{t}=\alpha_{0}+\sum_{i=1}^{p} \alpha_{i} r_{t-1}+\sum_{j=1}^{q} \beta_{j} h_{t-j}$.

Although the conditional distribution of the GARCH process has normal tails, the unconditional distribu-

\footnotetext{
$27 \mathrm{ARCH}$ and $\mathrm{GARCH}$ refer to Autoregressive Conditional Heteroscedasticity and Generalized Autoregressive Conditional Heteroscedasticity, respectively.
}

tion has some excess kurtosis. However, this may not be sufficient for modeling fat-tailed distributions since the tails of the unconditional distribution decay exponentially fast. In these cases, GARCH- $t$ (GARCH with Student's $t$-innovations) model may be an alternative. A weakness of the GARCH models is that they generally produce highly volatile quantile estimates (see Gençay, Selçuk, and Ulugülyağcı (2003)). Excessive volatility of quantile estimates is not desirable in risk management as it is costly to adjust the required capital frequently in light of the estimated VaR and difficult to regulate.

\subsection{Historical simulation}

The historical simulation method estimates the quantiles of an underlying distribution from the realization of the distribution. The VaR in this case is estimated by

$\operatorname{VaR}_{t}(\alpha)=F^{-1}(\alpha) r$

where $F^{-1}(\alpha) r$ is the $q$ th quantile $(q=1-\alpha)$ of the sample distribution.

The problem with this approach is that the empirical distribution function is not one-to-one but constant between two realizations. That is, we may not have observations corresponding to certain quantiles of the underlying distribution. A simple solution may be rounding the probability level to the nearest empirical probability and then taking the corresponding quantile as the desired quantile estimate. A more appropriate solution is to smooth the empirical distribution function with piecewise linear interpolation or kernel interpolation so that it is one-to-one.

The historical simulation method may fit the sample well, around the moderate quantiles, since no parametric form for the distribution is assumed. The disadvantage of this method is that the high quantile estimates are not reliable since they are calculated from only a few observations. Furthermore, it is not possible to obtain any quantile estimates above the highest observed quantile.

\subsection{VaR with EVT}

After estimating the shape and scale parameters $\xi$ and $\sigma$ with the maximum likelihood method, the EVT 
can be utilized to obtain a VaR estimate. For the maximum likelihood estimation, the density $f$ of the GPD distribution with parameters $\xi$ and $\sigma$ is (Smith, 1987)

$f(x)=\frac{1}{\sigma}\left(1+\xi \frac{x}{\sigma}\right)^{-\frac{1}{\xi}-1}$.

The corresponding log-likelihood function is

$\ell(\xi, \sigma)=-n \log (\sigma)-\left(\frac{1}{\xi}+1\right) \sum_{i=1}^{n} \log \left(1+\frac{\xi}{\sigma} X_{i}\right)$

where $n$ is the sample size. For $\xi>-0.5$, Hosking and Wallis (1987) present evidence that maximum likelihood regularity conditions are fulfilled and the maximum likelihood estimates are asymptotically normally distributed. Therefore, the approximate standard errors for the estimators of $\sigma$ and $\xi$ can also be obtained through maximum likelihood estimation.

For a given probability $q>F(u)$, an estimate of the VaR may be calculated by inverting the tail estimate in Eq. (6) to obtain ${ }^{28}$

$\operatorname{VaR}_{t}(\alpha)=u+\frac{\hat{\sigma}}{\hat{\xi}}\left[\left(\frac{n}{n_{u}} \alpha\right)^{-\hat{\xi}}-1\right]$

where $u$ is a threshold, $\hat{\sigma}$ is the estimated scale parameter, is the estimated shape parameter, $n$ is the sample size, $n_{u}$ is the number of exceedances and $\alpha=1-q .^{29}$

\subsection{Relative performance of VaR models}

We consider six different models for estimating one period ahead return predictions in both tails of the return distribution at different tail quantiles. These models are variance-covariance approach with normal distribution, variance-covariance approach with Student's $t$ distribution, historical simulation, adaptive GPD and nonadaptive GPD. In our preliminary anal-

\footnotetext{
28 Also, see Embrechts et al. (1997, p. 354) and McNeil (1999).

29 As an example, suppose that in daily stock returns, the threshold is determined as $6 \%$ and estimated parameters are $\hat{\sigma}=0.05$ and $\xi=0.50$. Further suppose that $n=1000$ and $n_{u}=50$. The VaR at $1 \%$ is $\operatorname{VaR}_{t}(0.01)=0.06+0.05 / 0.50\left[((1000 / 50) 0.01)^{-0.50}-1\right]$ $=0.184$. That is, the stock return will not exceed $18.4 \%$ in 1 day $99 \%$ of the time.
}

ysis, we also considered GARCH and GARCH varieties. However, as reported in Gençay et al. (2003), one period ahead estimated returns from GARCH models were extremely volatile without having any significant gain over other models in terms of forecast precision. Also, our GPD estimates indicated that starting from the second moment, certain moments of the return distribution may not exist. Therefore, we excluded the GARCH model from our relative performance study.

Our forecasting methodology is such that we adopt a sliding window with three different sizes: 500, 1000 and 1500 days. For example, with a window size of 500 , the window is placed between the 1 st and the 500th data points, the model is estimated and the return forecast is obtained for the 501st day at different quantiles. Next, the window is moved one period ahead to 2 nd and 501 st data points to obtain a forecast of the 502nd day return with updated parameters from this new sample. The last approach, nonadaptive GPD, does not utilize a window and uses all available data starting at the 500th, 1000th or 1500th day. For instance, the model is estimated using data from the first 500 days, then the 501st day return is estimated and stored. Next, the model is estimated adding the 501st day return into the sample and a forecast of the 502nd day return is obtained and stored.

There is no difficulty in obtaining forecasts from the variance-covariance approach with normal distribution since it requires only the sample mean and the sample standard deviation. However, other modeling approaches require certain assumptions. It is practically impossible to determine a best parameterization or a threshold value for each window size in other modeling approaches. After a preliminary search, we decided to use a Student's $t$ distribution with 6 degrees of freedom for the variance-covariance approach with Student's $t$ distribution. Similarly, instead of determining a threshold value at each step, we utilized the upper $2.5 \%$ of the ranked sample in both adaptive and nonadaptive GPD approaches. For the historical simulation, piecewise linear interpolation is chosen for missing values at certain quantiles.

The relative performance of each model is summarized by a "violation ratio". A violation occurs if the realized return is greater than the estimated one in a given day. The violation ratio is defined as the total 
number of violations, divided by the total number of one step ahead forecasts. The motivation behind this measure is as follows. When we make a forecast of the VaR at a certain quantile $q$, we expect that the realized return will be higher $(1-q)$ percent of the time if the model is correct. In other words, the expected violation ratio at $q$ th percentile is $(1-q)$. For example, expected violation ratio is $5 \%$ at the 95th quantile. A violation ratio higher than the expected one indicates that the model consistently underestimates the return (=risk) at the tail. This is because the realized returns were higher than the model's prediction, resulting in a violation ratio higher than the expected one. On the other hand, a violation ratio less than the expected one at a given quantile implies that the model consistently overestimates the return at that quantile. For example, suppose that the violation ratio is zero percent at the 95th quantile. This means that all realized returns were always less than the model's prediction. However, we expect that the true model should result in 5\% violation ratio at this particular quantile.

It is tempting to think that a small violation ratio is preferable at a given quantile. However, a small violation ratio (smaller than the expected one at a given quantile) may or may not be desirable. As mentioned above, a smaller violation ratio indicates an overestimation of the risk. From a risk management point of view, estimated return at the right tail determines the amount of capital that should be allocated to cover the possible loss (assuming a short position in the market). Therefore, a smaller violation ratio (consistent overestimation of the return) signals an excessive capital allocation (more than necessary) and the portfolio holder registers a loss of interest rate income. However, this might be preferable for regulatory purposes since a regulatory body is only interested in an adequate amount of capital in case of excessive losses. On the other hand, a large violation ratio (consistent underestimation of the risk) results in less required capital allocation. If the whole purpose of VaR estimation is to meet the regulatory requirement, a model with large violation ratio is preferable from a financial institution's point of view.

In practice, one hardly knows whether a model will underpredict or overpredict the risk. If the preference is a model that underpredicts the risk for the reasons mentioned above (less capital allocation for regulatory purposes), and if all the models under consideration are overpredicting it, a model with least overprediction is preferable. That is, a model with a violation ratio close to the expected violation ratio from below is chosen. For example, if there are three models with $3 \%, 4 \%$ and $4.5 \%$ violation ratio at the 95 th quantile, the one with $4.5 \%$ is preferable since it signals less capital allocation than others by generating lower return forecasts at that quantile. On the other hand, if the preference is a model that overpredicts the risk (forcing financial institutions to allocate more capital), and if all the models underpredict it, a model with a violation ratio close to the expected one from above is preferable. For example, if there are three models with $5.5 \%, 6 \%$ and $7 \%$ violation ratio at the 95 th quantile, the one with $5.5 \%$ is preferable since it signals more capital allocation than others by generating higher return forecasts at that quantile.

Table 5 illustrates which models perform best in terms of the least underestimation of the risk at

Table 5

Models with least underestimation of the risk at different quantiles in different markets

\begin{tabular}{|c|c|c|c|c|c|c|c|c|c|c|}
\hline & \multicolumn{5}{|c|}{ Left Tail } & \multicolumn{5}{|c|}{ Right Tail } \\
\hline & $5 \%$ & $2.5^{\circ}$ & $61 \%$ & $0.5 \%$ & $0.1 \%$ & $5 \%$ & $2.5 \%$ & $1 \%$ & $0.5 \%$ & $0.1 \%$ \\
\hline Argentina & $\mathrm{H}$ & $\mathrm{N}$ & $\mathrm{T}$ & $E$ & $E$ & $E$ & $\mathrm{~N}$ & $\mathrm{~T}$ & $E$ & E \\
\hline Brazil & $\mathrm{E}$ & $\mathrm{T}$ & $E$ & E & $\mathrm{E}$ & $E$ & $\mathrm{H}$ & $\mathrm{H}$ & $\mathrm{T}$ & E \\
\hline Hong Kong & $\mathrm{H}$ & E & E & $\mathrm{E}$ & $\mathrm{E}$ & E & E & E & $T$ & $\mathrm{~T}$ \\
\hline Indonesia & $\mathrm{T}$ & $\mathrm{T}$ & E & E & E & $\mathrm{N}$ & $\mathrm{N}$ & $\mathrm{N}$ & $\mathrm{T}$ & E \\
\hline Korea & $\mathrm{T}$ & $\mathrm{T}$ & $\mathrm{T}$ & $\mathrm{T}$ & $\mathrm{T}$ & E & $\mathrm{T}$ & $E$ & E & E \\
\hline Mexico & $\mathrm{N}$ & $E$ & $\mathrm{~T}$ & $\mathrm{~T}$ & $\mathrm{E}$ & $\mathrm{N}$ & $\mathrm{T}$ & $\mathrm{T}$ & $\mathrm{T}$ & $\mathrm{T}$ \\
\hline Singapore & $\mathrm{E}$ & $\mathrm{N}$ & E & E & E & $E$ & $\mathrm{~N}$ & $\mathrm{~T}$ & $\mathrm{~T}$ & E \\
\hline Taiwan & $\mathrm{N}$ & $\mathrm{T}$ & $\mathrm{T}$ & $\mathrm{T}$ & E & $\mathrm{N}$ & E & $\mathrm{T}$ & E & E \\
\hline Turkey & $\mathrm{T}$ & $\mathrm{T}$ & E & E & $\mathrm{E}$ & $\mathrm{H}$ & E & $\mathrm{T}$ & E & $\mathrm{E}$ \\
\hline
\end{tabular}

At a given quantile, we consider only those models that produce an underestimate of the risk according to the VaR violation ratios. For example, at $95 \%$ quantile ( $5 \%$ at the tail), the expected value of VaR violation ratio is $5 \%$. A model with a VaR violation ratio greater than 5 underestimates the risk (produces low return forecasts) while a value less than 5 implies overestimation (large return forecasts). We ranked the models that underestimate the risk according to how close they are to the expected value. If, for instance, the estimated VaR violation ratios are $5.1 \%, 5.5 \%$ and $6.0 \%$ from three different models at the $5 \%$ tail, the model with $5.1 \%$ violation ratio is selected as the best model in terms of least underestimation of the risk for that tail value. If there is a tie, a model with the second best performance is selected. T: Student's $t$ distribution, N: Normal distribution, H: Historical simulation, E: Generalized Pareto Distribution (GPD). Notice that the GPD based models perform better as one moves further in the tail. 
different quantiles at both tails of the return distributions in different emerging markets. At a given quantile, we consider only those models that produce an underestimate of the risk according to the VaR violation ratios. These models are ranked according to how close they are to the expected value. If, for instance, the estimated $\mathrm{VaR}$ violation ratios are $5.5 \%, 5.3 \%$ and $5.1 \%$ from three different models, the model with the $5.1 \%$ violation ratio is selected as the best model in terms of least underestimation of the risk for the 95th quantile. If there is a tie between two models, the one with the second best performance is chosen. At moderate levels of both tails, there is no clear winner. However, as we move towards the higher quantiles such as 99th, 995th and 999th, the GPD model clearly outperforms others.

In a similar fashion, Table 6 illustrates the best model in terms of least overestimation of the risk. Here, we consider only those models that produce an overestimate of the risk according to the VaR violation ratios at a given quantile. The models that overestimate the risk are ranked according to how close they

Table 6

Models with least overestimation of the risk at different quantiles in different markets

\begin{tabular}{|c|c|c|c|c|c|c|c|c|c|c|}
\hline & \multicolumn{5}{|c|}{ Left Tail } & \multicolumn{5}{|c|}{ Right Tail } \\
\hline & $5 \%$ & $2.5^{\circ}$ & $\% 1 \%$ & $0.5 \%$ & $0.1 \%$ & $5 \%$ & $2.5 \%$ & $1 \%$ & $0.5 \%$ & $0.1 \%$ \\
\hline Argentina & $\mathrm{N}$ & $\mathrm{T}$ & E & $\mathrm{E}$ & $\mathrm{E}$ & $E$ & E & E & E & E \\
\hline Brazil & $\mathrm{E}$ & $\mathrm{E}$ & E & $\mathrm{E}$ & $\mathrm{E}$ & $\mathrm{E}$ & $\mathrm{H}$ & $\mathrm{T}$ & $\mathrm{T}$ & E \\
\hline Hong Kong & $\mathrm{N}$ & $\mathrm{T}$ & E & E & $E$ & E & $\mathrm{N}$ & $\mathrm{T}$ & $\mathrm{T}$ & E \\
\hline Indonesia & $\mathrm{N}$ & $\mathrm{T}$ & E & E & E & $\mathrm{T}$ & $\mathrm{T}$ & $\mathrm{T}$ & $\mathrm{N}$ & $\mathrm{T}$ \\
\hline Korea & $\mathrm{T}$ & $\mathrm{T}$ & $\mathrm{T}$ & $\mathrm{T}$ & $\mathrm{T}$ & $\mathrm{T}$ & $\mathrm{T}$ & E & E & E \\
\hline Mexico & $\mathrm{T}$ & $\mathrm{T}$ & $\mathrm{T}$ & E & E & E & $\mathrm{T}$ & $\mathrm{T}$ & E & $E$ \\
\hline Singapore & E & $\mathrm{T}$ & $\mathrm{T}$ & E & $\mathrm{H}$ & $\mathrm{N}$ & $\mathrm{N}$ & $\mathrm{T}$ & E & $\mathrm{E}$ \\
\hline Taiwan & $\mathrm{N}$ & $\mathrm{T}$ & $\mathrm{T}$ & $\mathrm{T}$ & $\mathrm{E}$ & $\mathrm{N}$ & E & $\mathrm{T}$ & E & E \\
\hline Turkey & $\mathrm{T}$ & $\mathrm{T}$ & E & E & E & $\mathrm{N}$ & $\mathrm{T}$ & $\mathrm{E}$ & $\mathrm{T}$ & E \\
\hline
\end{tabular}

At a given quantile, we consider only those models that produce an exact or an overestimate of the risk according to the VaR violation ratios. For example, at $95 \%$ quantile ( $5 \%$ at the tail), the expected value of VaR violation ratio is $5 \%$. A model with a VaR violation ratio greater than 5 underestimates the risk while a value less than 5 implies overestimation. We ranked the models that overestimate the risk according to how close they are to the expected value. Suppose that at $5 \%$ tail, the estimated $\mathrm{VaR}$ violation ratios are $4.5 \%, 3.5 \%$ and $1 \%$ from three different models. The model with $4.5 \%$ violation ratio is selected as the best model for that tail value. If there is a tie, a model with the second best performance is selected. T: Student's $t$ distribution, N: Normal distribution, H: Historical simulation, E: Generalized Pareto Distribution (GPD). Notice that the GPD based models perform better as one moves further in the tail.
Table 7

Models with least distance to the expected VaR violation ratios

\begin{tabular}{|c|c|c|c|c|c|c|c|c|c|c|}
\hline & \multicolumn{5}{|c|}{ Left Tail } & \multicolumn{5}{|c|}{ Right Tail } \\
\hline & $5 \%$ & $2.5 \%$ & $1 \%$ & $0.5 \%$ & $0.1 \%$ & $5 \%$ & $2.5 \%$ & $1 \%$ & $0.5 \%$ & $0.1 \%$ \\
\hline Argentina & $\mathrm{H}$ & $\mathrm{N}$ & E & E & E & E & $\mathrm{N}$ & $\mathrm{T}$ & E & E \\
\hline Brazil & $\mathrm{E}$ & $\mathrm{H}$ & E & $\mathrm{E}$ & $\mathrm{E}$ & E & $\mathrm{H}$ & $\mathrm{H}$ & $\mathrm{T}$ & E \\
\hline Hong Kong & $\mathrm{H}$ & $\mathrm{T}$ & E & E & E & E & $\mathrm{N}$ & $\mathrm{T}$ & $\mathrm{T}$ & $\mathrm{T}$ \\
\hline Indonesia & $\mathrm{N}$ & $\mathrm{T}$ & E & $\mathrm{H}$ & E & $\mathrm{T}$ & $\mathrm{T}$ & $\mathrm{N}$ & $\mathrm{N}$ & $\mathrm{E}$ \\
\hline Korea & $\mathrm{T}$ & $\mathrm{T}$ & $\mathrm{T}$ & $\mathrm{T}$ & $\mathrm{T}$ & E & $\mathrm{T}$ & E & E & $\mathrm{E}$ \\
\hline Mexico & $\mathrm{N}$ & $\mathrm{T}$ & $\mathrm{T}$ & $\mathrm{E}$ & E & E & $\mathrm{T}$ & $\mathrm{T}$ & E & $\mathrm{E}$ \\
\hline Singapore & $\mathrm{E}$ & $\mathrm{N}$ & E & E & E & $\mathrm{N}$ & $\mathrm{N}$ & $E$ & E & $E$ \\
\hline Taiwan & $\mathrm{N}$ & $\mathrm{T}$ & $\mathrm{T}$ & $\mathrm{T}$ & $\mathrm{E}$ & $\mathrm{N}$ & E & $\mathrm{T}$ & E & $\mathrm{E}$ \\
\hline Turkey & $\mathrm{T}$ & $\mathrm{T}$ & $\mathrm{E}$ & E & E & $\mathrm{H}$ & $\mathrm{T}$ & $\mathrm{T}$ & E & $\mathrm{E}$ \\
\hline
\end{tabular}

For example, at $95 \%$ quantile ( $5 \%$ at the tail), the expected value of $\mathrm{VaR}$ violation ratio is 5 percent. A model with a VaR violation ratio closest to 5 is picked up as the best model. Suppose that at $5 \%$ tail, the estimated VaR violation ratios are $4.8 \%, 5.3 \%$ and $4.3 \%$ from three different models. The model with $4.8 \%$ violation ratio is selected as the best model for that tail value. If there is a tie, the model with a closer second VaR ratio is selected. T: Student's $t$ distribution, N: Normal distribution, H: Historical simulation, E: Generalized Pareto Distribution (GPD). Notice that the GPD based models perform better as one moves further in the tail.

are to the expected value. Suppose that at the $5 \%$ tail, the estimated VaR violation ratios are $4.5 \%, 3.5 \%$ and $1 \%$ from three different models. The model with a $4.5 \%$ violation ratio is selected as the best model for that tail value since it is the one with least overestimation. If there is a tie, the model with the second best violation ratio is selected. Again, at moderate levels of both tails, there is no clear winner. However, as we move towards the higher quantiles, the GPD model is clearly better. ${ }^{30}$

Finally, Table 7 provides an overall picture of relative performance of different models at different quantiles at both tails of the return distributions. In this table, a model which produces a violation ratio with least distance to the expected violation ratio at a given quantile is picked up as the best model. Especially at 99th and higher quantiles, the GPD model clearly dominates others in terms of VaR forecasting in nine emerging markets we cover in this study. We conclude that the GPD and the extreme value theory is an indispensable part of risk management, especially in the VaR calculations and stress testing.

\footnotetext{
${ }^{30}$ Forecasting results in terms of violation ratios with different sample sizes at different quantiles for each country are not reported due to space limitations. The results are available from the authors upon request.
} 


\section{Conclusions}

We investigated the nonlinear estimation and forecasting of extreme values of daily stock market returns in emerging markets. It is shown that the Generalized Pareto Distribution (GPD) fits the tails of the return distributions in these markets well. The results indicate that the daily return distributions have different characteristics at left and right tails. Therefore, the risk and reward are not equally likely in these economies. Estimates of left and right tails at 0.999 percentile along with $95 \%$ confidence intervals show that it is possible to observe over $10 \%$ loses in 1 day in all emerging markets, whereas the lower confidence intervals for the positive returns are less than $10 \%$, except in Brazil and Turkey. We also investigated the relative performance of each model in terms of VaR forecasting in a dynamic setting. Especially at 99th and higher quantiles, the GPD model clearly dominates others in terms of VaR forecasting. We conclude that the GPD and the extreme value theory are an indispensable part of risk management in general and the VaR calculations in particular, in emerging markets.

Emerging market economies are fundamentally different than developed economies in terms of changes in dynamic structure of the economy. In other words, these economies are much more subject to regime switches in short periods of time. Therefore, it is quite possible that the underlying probability distributions of certain variables or the parameters of the existing distributions change quite often. Any modeling exercise in these economies should take into account this fact and incorporate any changes in the environment into the modeling. The sliding window approach in this paper aims at capturing the changing dynamics in the economy.

There are possible directions for future research. The daily $\log$ returns are calculated in terms of domestic currency in this study. An international portfolio holder might be interested in US dollar (or some other currency) returns. Converting the domestic currency returns into another stable currency would make the returns comparable among different economies. It would also (partially) eliminate the effect of high inflation on the rate of returns. However, an analysis of stock market returns in dollar terms combines the dynamics of the stock return in the economy and the exchange rate and this complicates the analysis. A multivariate approach, as in Hauksson et al. (2000), should be adopted to have a complete picture of the risk and reward in the emerging markets.

\section{Acknowledgements}

Ramazan Gençay gratefully acknowledges financial support from the Swiss National Science Foundation under NCCR-FINRISK, Natural Sciences and Engineering Research Council of Canada and the Social Sciences and Humanities Research Council of Canada.

We thank Abdurrahman Ulugülyağci for research assistance. We are grateful to the co-editors of this special issue and the anonymous referee for their valuable suggestions.

Faruk Selçuk greatly acknowledges financial support from the Colorado College Social Science Executive Division Research and Development Fund.

\section{References}

Balkema, A. A., \& de Haan, L. (1974). Residual lifetime at great age. Annals of Probability, 2, 792-804.

Basel (1996). Overview of the amendment to the capital accord to incorporate market risk. Basel: Basel Committee on Banking Supervision.

Bollerslev, T. (1986). Generalized autoregressive conditional heteroscedasticity. Journal of Econometrics, 31, 307-327.

Boothe, P., \& Glassman, P. D. (1987). The statistical distribution of exchange rates. Journal of International Economics, 22, 297-319.

Corsetti, G., Pesenti, P., \& Roubini, N. (1999). What caused the Asian currency and financial crisis. Japan and World Economy, 11, 305-373.

Dacorogna, M. M., Gençay, R., Müller, U. A., Olsen, R. B., \& Pictet, O. V. (2001). An introduction to high-frequency finance. San Diego: Academic Press.

Dacorogna, M. M., Pictet, O. V., Müller, U. A., \& de Vries, C. G. (2001). Extremal forex returns in extremely large datasets. Extremes 4, 105-127.

Danielsson, J., de Haan, L., Pend, L., \& de Vries, C. G. (2001). Using a bootstrap method to choose the sample fraction in tail index estimation. Journal of Multivariate Analysis, 76, $226-248$.

Danielsson, J., \& de Vries, C. G. (1997). Tail index and quantile estimation with very high frequency data. Journal of Empirical Finance, 4, 241-257. 
de Haan, L. (1990). Fighting the Arch-enemy with mathematics. Statistica Neerlandica, 44, 45-68.

de Haan, L., Jansen, D. W., Koedijk, K. G., \& de Vries, C. G. (1994). Safety first portfolio selection, extreme value theory and long run asset risks. In J. Galambos, J. Lechner, \& E. Simiu (Eds.), Extreme value theory and applications (pp. 471-488). Dordrecht: Kluwer.

Deheuvels, P., Hausler, E., \& Mason, D. M. (1988). Almost sure convergence of the Hill estimator. Mathematical Proceeding of Cambridge Philosophical Society, 104, 371-381.

Diaz, A. C. (1984). Latin American debt: I don't think we are in Kansas anymore. Brookings Papers on Economic Activity, 2, $335-389$.

Dornbush, R. (2001). A primer on emerging market crisis. Boston, MA: Department of Economics, M.I.T.

Dowd, K. (1998). Beyond Value-at-Risk: The new science of risk management. Chichester: Wiley.

Duffie, D., \& Pan, J. (1997). An overview of Value-at-Risk. Journal of Derivatives, 7, 7-49.

Embrechts, P. (1999). Extreme value theory in finance and insurance. Manuscript. Zurich, Switzerland: Department of Mathematics, ETH, Swiss Federal Technical University.

Embrechts, P. (2000a). Extreme value theory: Potentials and limitations as an integrated risk management tool. Manuscript. Zurich, Switzerland: Department of Mathematics, ETH, Swiss Federal Technical University.

Embrechts, P. (2000b). Extremes and integrated risk management. London: Risk Books and UBS Warburg.

Embrechts, P., Kluppelberg, C., \& Mikosch, C. (1997). Modeling extremal events for insurance and finance. Berlin: Springer.

Embrechts, P., Resnick, S., \& Samorodnitsky, G. (1998). Extreme value theory as a risk management tool. Manuscript. Zurich, Switzerland: Department of Mathematics, ETH, Swiss Federal Technical University.

Engle, R. F. (1982). Autoregressive conditional heteroscedastic models with estimates of the variance of United Kingdom inflation. Econometrica, 50, 987-1007.

Falk, M., Hüssler, J., \& Reiss, R. (1994). Laws of small numbers: Extremes and rare events. Basel: Birkhäuser.

Fisher, R. A., \& Tippett, L. H. C. (1928). Limiting forms of the frequency distribution of the largest or smallest member of a sample. Proceeding of Cambridge Philosophical Society, 24, 180-190.

Gençay, R., Selçuk, F., \& Ulugülyağcı, A. (2001). EVIM: A software package for extreme value analysis in Matlab. Studies in Nonlinear Dynamics and Econometrics, 5, 213-239.

Gençay, R., Selçuk, F., \& Ulugülyağcı, A. (2003). High volatility, thick tails and extreme value theory in Value-atRisk estimations. Insurance: Mathematics and Economics (forthcoming).

Ghose, D., \& Kroner, K. F. (1995). The relationship between GARCH and symmetric stable distributions: finding the source of fat tails in the financial data. Journal of Empirical Finance, 2, $225-251$.

Gnedenko, B. V. (1943). Sur la distribution limite du terme d'une serie aleatoire. Annals of Mathematics, 44, 423-453.

Goldie, C. M., \& Smith, R. L. (1987). Slow variation with remain- der: theory and applications. Quarterly Journal of Mathematics, Oxford 2nd Series, 38, 45-71.

Hall, P. (1982). On some simple estimates of an exponent of regular variation. Journal of the Royal Statistical Society, Series B, 44, $37-42$.

Hauksson, H. A., Dacorogna, M., Domenig, T., Müller, U., \& Samorodnitsky, G. (2000). Multivariate extremes, aggregation and risk estimation. Manuscript. Ithaca, NY: School of Operations Research and Industrial Engineering, Cornell University.

Hill, B. M. (1975). A simple general approach to inference about the tail of a distribution. Annals of Statistics, 3, 1163-1174.

Hols, M. C., \& de Vries, C. G. (1991). The limiting distribution of extremal exchange rate returns. Journal of Applied Econometrics, 6, 287-302.

Hosking, J. R. M., \& Wallis, J. R. (1987). Parameter and quantile estimation for the generalized Pareto distribution. Technometrics, 29, 339-349.

Jenkinson, A. F. (1955). Distribution of the annual maximum (or minimum) values of meteorological elements. Quarterly Journal of Royal Meteorological Society, 81, 145-158.

Jorion, P. (1997). Value-at-Risk: The new benchmark for controlling market risk. Chicago: McGraw-Hill.

Koedijk, K. G., Schafgans, M. M. A., \& de Vries, C. G. (1990). The tail index of exchange rate returns. Journal of International Economics, 29, 93-108.

Leadbetter, M., Lindgren, G., \& Rootzén, H. (1983). Extremes and related properties of random sequences and processes. Springer series in statistics. New York: Springer-Verlag.

Levich, R. M. (1985). Empirical studies of exchange rates: Price behavior, rate determination and market efficiency. Handbook of Economics, 6, 287-302.

Loretan, M., \& Phillips, P. C. B. (1994). Testing the covariance stationary of heavy-tailed time series. Journal of Empirical Finance, 1, 211-248.

Mandelbrot, B. (1963a). New methods in statistical economics. Journal of Political Economy, 71, 421-440.

Mandelbrot, B. (1963b). The variation of certain speculative prices. Journal of Business, 36, 394-419.

Mandelbrot, B. B. (2001). Scaling in financial prices: IV. Multifractal concentration. Quantitative Finance, 1, 641-649.

Mason, D. M. (1982). Laws of large numbers for sums of extreme values. Annals of Probability, 10, 756-764.

McNeil, A. J. (1997). Estimating the tails of loss severity distributions using extreme value theory. ASTIN Bulletin, 27, $1117-1137$.

McNeil, A. J. (1998). Calculating quantile risk measures for financial time series using extreme value theory. Manuscript. Zurich, Switzerland: Department of Mathematics, ETH, Swiss Federal Technical University.

McNeil, A.J. (1999). Extreme value theory for risk managers. Internal modeling and CAD II, Risk books. London, UK, pp. 93-118.

McNeil, A. J., \& Frey, R. (2000). Estimation of tail-related risk measures for heteroscedastic financial time series: An extreme value approach. Journal of Empirical Finance, 7, $271-300$.

Müller, U. A., Dacorogna, M. M., \& Pictet, O. V. (1998). Heavy 
tails in high-frequency financial data. In R. J. Adler, R. E. Feldman, \& M. S. Taqqu (Eds.), A practical guide to heavy tails: Statistical techniques and applications (pp. 55-77). Boston: Birkhäuser.

Mussa, M. (1979). Empirical regularities in the behavior of exchange rates and theories of the foreign exchange market. Carnegie-Rochester Conference Series on Public Policy, 11, $9-57$.

Nelson, D. B. (1991). Conditional heteroscedasticity in asset returns: a new approach. Econometrica, 59, 347-370.

Pickands, J. (1975). Statistical inference using extreme order statistics. Annals of Statistics, 3, 119-131.

Pictet, O. V., Dacorogna, M. M., \& Müller, U. A. (1998). Hill, bootstrap and jackknife estimators for heavy tails. In Taqqu, M. (Ed.), A Practical Guide to Heavy Tails: Statistical Techniques and Applications (pp. 283-310). Boston, MA: Birkhäuser.

Reiss, R., \& Thomas, M. (1997). Statistical analysis of extreme values. Basel: Birkhäuser.

Rootzén, H., Leadbetter, M. R., \& de Haan, L. (1992). Tail and quantile estimators for strongly mixing stationary processes. Report. Chapel Hill, NC: Department of Statistics, University of North Carolina at Chapel Hill.

Smith, R. L. (1987). Estimating tails of probability distributions. Annals of Statistics, 15, 1174-1207.

Teugels, J. B. J., \& Vynckier, P. (1996). Practical analysis of extreme values. Leuven: Leuven Univ. Press.

von Mises, R. (1964). La distribution de la plus grande de $\mathrm{n}$ valeurs. Rev. Math. Union Interbalcanique, 1, 141-160 (Reproduced in selected papers of Richard von Mises);

American Mathematical Society, 2, 271-294.
Biographies: Professor Ramazan GENÇAY is in the Economics Department at the University of Windsor, Ontario, Canada. His areas of specialization are financial econometrics, nonlinear time series, nonparametric econometrics and chaotic dynamics. He has over 50 scientific publications, which appeared in finance, economics, engineering, statistics and physics journals. Some of his publications are published in the Journal of the American Statistical Association, Journal of Econometrics, Journal of International Economics, International Economic Review, Journal of Nonparametric Statistics, Journal of Empirical Finance, Journal of Economic Dynamics and Control, Journal of Applied Econometrics, European Economic Review, Journal of Forecasting, IEEE Transactions on Neural Networks, Physica A, Physica D and Physics Letters A. He is a co-author of two books, An Introduction to High-Frequency Finance and Wavelets and Other Filtering Methods in Finance and Economics published by Academic Press. Dr. Gencay is an associate editor of Studies in Nonlinear Dynamics and Econometrics and IEEE Transactions on Computational Finance.

Faruk SELÇUK is an Associate Professor at Bilkent University, Ankara, Turkey. He earned his PhD from The Graduate Center, City University of New York. His research interests are in financial econometrics and time series analysis, wavelets in finance and economics and emerging markets. His research has been published in leading journals including Journal of International Money and Finance, Quantitative Finance, Physica A, Studies in Nonlinear Dynamics and Econometrics and Economics Letters. He is a coauthor of An Introduction to Wavelets and Other Filtering Methods in Finance and Econometrics. 